\title{
Impact of an electronic alert on prescription patterns of meropenem, voriconazole and caspofungin
}

\author{
Lionel Chok ${ }^{*}{ }^{\dagger}$, Katharina Kusejko ${ }^{\dagger}$, Nadia Eberhard, Sandra E. Chaudron, Dirk Saleschus, Claudine Kocher,
} Roger D. Kouyos ${ }^{\dagger}$, Rainer Weber ${ }^{\dagger}$ and Stefan P. Kuster ${ }^{\dagger}$

\begin{abstract}
Background: Antimicrobial stewardship programs promote the appropriate use of antimicrobial substances through the implementation of evidence-based, active and passive interventions. We analyzed the effect of a computerassisted intervention on antimicrobial use in a tertiary care hospital.

Methods: Between 2011 and 2016 we introduced an electronic alert for patients being prescribed meropenem, voriconazole and caspofungin. At prescription and at day 3 of treatment, physicians were informed about the risk related to these antimicrobial substances by an electronic alert in the medical records. Physicians were invited to revoke or confirm the prescription and to contact the infectious disease (ID) team. Using interrupted time series regression, the days of therapy (DOTs) and the number of prescriptions before and after the intervention were compared.

Results: We counted 64,281 DOTs for 5549 prescriptions during 4100 hospital stays. Overall, the DOTs decreased continuously over time. An additional benefit of the alert could not be observed. Similarly, the number of prescriptions decreased over time, without significant effect of the intervention. When considering the three drugs separately, the alert impacted the duration (change in slope of DOTs/1000 bed days; $P=0.0017$ ) as well as the number of prescriptions (change in slope of prescriptions $/ 1000$ bed days; $P<0.001$ ) of voriconazole only.

Conclusions: The introduction of the alert lowered prescriptions of voriconazole only. Thus, self-stewardship alone seems to have a limited impact on electronic prescriptions of anti-infective substances. Additional measures such as face-to-face prompting with ID physicians or audit and feedback are indispensable to optimize antimicrobial use.
\end{abstract}

Keywords: Antimicrobial stewardship, Antimicrobial consumption, Antimicrobial prescriptions, Antibiotic consumption, Electronic alert, Self-stewardship

\section{Background}

Antibiotic overuse in human and veterinary medicine has led to an increase in resistant bacteria [1-4]. Antimicrobial stewardship programs (ASPs) promote the optimal use of anti-infective drugs, reduce unnecessary

\footnotetext{
*Correspondence: lionel.chok@bluewin.ch

†Lionel Chok, Katharina Kusejko, Roger D. Kouyos, Rainer Weber, Stefan P. Kuster contributed equally to this work

Division of Infectious Diseases and Hospital Epidemiology, University

Hospital and University of Zurich, Rämistrasse 100, Zurich, Switzerland
}

antimicrobial prescriptions, resistance pressure in the hospital, drug-related side effects and improve patient outcomes [5-8]. ASPs consist of active, usually prospective audits with patient-based interventions and passive interventions, including non-patient-based educational measures and hospital guidelines $[9,10]$. Passive strategies seem to be most effective when combined with active interventions. Interventions may be drug-based or disease-based. 
Resources allocated to ASPs may often be limited. Individual disease-based support for antimicrobial decision making by an infectious disease (ID) specialist or a pharmacist with antimicrobial stewardship training is timeconsuming and costly. Computer-based surveillance and clinical decision support systems have been shown to improve the use of antimicrobials and thus may be part of ASPs [9]. Algorithms providing assistance on antibiotic decision-making may thus allow a broad and cost-saving implementation of ASP interventions.

The Guidelines of the Infectious Diseases Society of America (IDSA) and the Society for Healthcare Epidemiology of America (SHEA) on ASPs support interventions encouraging routine review of the appropriateness of antibiotic therapy by the prescriber itself [8].

Similar to trends observed in Europe, consumption of carbapenems in Swiss hospitals increased by almost $85 \%$ between 2004 and 2010 [11]. The emergence of carbapenem-resistant Enterobacterales is thought to be strongly linked to antibiotic overuse and the resulting selection pressure in humans, apart from insufficient infection control measures and antibiotic consumption in agriculture [12-15].

On July 1st, 2011, the Department of Infectious Diseases at the University Hospital Zurich implemented a computer-assisted drug-based intervention on all acute care units which aimed to optimize the use of the broadspectrum and costly antimicrobials meropenem, voriconazole and caspofungin. Meropenem is a last resort substance and had the broadest spectrum of activity at the time. It accounted for $29 \%$ of the total antibiotic cost and was the third most used antibiotic (4.63 defined daily doses (DDD)/100 bed days; ranked after amoxicillin/clavulanic acid and ciprofloxacin, but before piperacillin/tazobactam; non-published data) in our institution in 2009. Caspofungin and voriconazole accounted for $25 \%$ and respectively $6 \%$ of the total anti-infective costs but only for $2 \%$ and $1 \%$ of the anti-infective use, respectively, in our institution in 2009. During the electronic prescription of one of these substances, a pop-up window appeared, which highlighted the restrictions and indications associated with these antimicrobials.

The objectives of this study were to assess the effect of the electronic reminder on the number of days of therapy (DOTs) of meropenem, caspofungin and voriconazole, and whether the number of prescriptions of these antimicrobials was affected by the intervention, respectively.

\section{Methods}

\section{Study design, setting and ethical considerations}

The prospective, single-centre, before-and-after study was conducted at the University Hospital Zurich, a reference centre for 1.5 million inhabitants and one of the largest Swiss tertiary care hospitals with 950 beds. The Department of Infectious Diseases and Hospital Epidemiology designed and planned the intervention. The Medical Data Management Team implemented the alert in the electronic patient charts $\left(\operatorname{KISIM}^{\mathrm{TM}}, \mathrm{Cistec}^{\circledR}\right.$, Switzerland).

This study complies with the national legal and regulatory requirements and the current version of the Declaration of Helsinki and has been approved by the Canton Ethics Committee (Kantonale Ethikkommission Zurich, Switzerland, KEK-ZH-Nr 2011-0031/1 and PB_2018-00032).

\section{Intervention}

The intervention consisted of the appearance of an alert in the patient's electronic chart in case of prescriptions of meropenem, voriconazole or caspofungin (Fig. 1).

A pop-up window appeared for prescriptions in all acute care units from July 1,2011 , to June 30,2016 , and the following text was displayed: "Prescription of meropenem or new antifungal substances. You are prescribing voriconazole, caspofungin or meropenem. Because of the risk of antibiotic resistance (meropenem) or their high costs (antifungals), the use of these substances at the University Hospital Zurich is restricted. Is there an indication according to the hospital antibiotic guidelines? [Link to the guidelines provided]". The physician then had to select "confirm prescription" or "cancel/stop prescription". If prescription was confirmed, a horizontal bar appeared in the warning/comment section of the electronic patient chart $72 \mathrm{~h}$ after the prescription, displaying the following text: "Comment on prescription of antiinfectives. The patient has been prescribed voriconazole, caspofungin or meropenem for $72 \mathrm{~h}$. According to the clinical course and the results, please assess whether (1) the current therapy should be continued, (2) the therapy may be streamlined (e.g. narrower spectrum) or (3) the therapy may be stopped. We advise you to involve the ID consultation service if you continue the current therapy (telephone contact provided)". The bar was displayed in orange colour, indicating in our system that this information had to be validated by the treating physician, and turned into green colour if the physician confirmed having read the information.

\section{Data}

Based on the Anatomical Therapeutic Chemical (ATC) Classification System, prescriptions of antibiotic groups ATC J01DH02 (meropenem, parenteral route) and antifungal groups J02AC03 (voriconazole, parenteral and oral) and J02AX04 (caspofungin, parenteral) for inpatients on acute wards (excluding intensive care units) 
Prescription of meropenem, voriconazole or caspofungin on acute care units of the University Hospital Zurich (July 1, 2011-June 30, 2016)

Apparition of this message in a pop-up window:

"Prescription of meropenem or new antifungal substances. You are prescribing voriconazole, caspofungin or meropenem. Because of the risk of antibiotic resistance (meropenem) or their high costs (antifungals), the use of these substances at the University Hospital Zurich is restricted. Is there an indication according to the hospital antibiotic guidelines [Electronic link to the guidelines] ?"

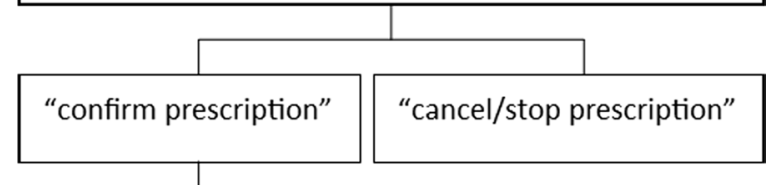

$72 \mathrm{~h}$ hours after the prescription, the following message appeared in orange in the patient chart:

"Comment on prescription of anti-infectives. The patient has been prescribed voriconazole, caspofungin or meropenem for 72 hours. According to the clinical course and the results, please assess whether 1 . the current therapy should be continued, 2 . the therapy may be streamlined (e.g. narrower spectrum) or 3 . the therapy may be stopped. We advise you to involve the ID consultation service if you continue the current therapy (telephone contact provided)".

Message turned into green colour if the physician confirmed having read the information.

Fig. 1 Intervention flow chart

were analyzed [16]. Antimicrobial prescriptions from July 1, 2010 to June 30, 2016 were included in the analyses.

The IT Department retrieved prescription data from electronic medical records. Prescription data contained (i) brand name, substance name, administration route and dose per unit of prescriptions of the substances meropenem, voriconazole and caspofungin, (ii) start and end of the prescription, (iii) birth date and age (in years) at admission of the patients, (iv) date of hospital admission and discharge and (v) date of admission to and discharge from the ward where the anti-infective substance was prescribed. We retrieved gender for each patient and number of bed days and number of admissions of all patients for each unit from the SAP ${ }^{\circledR}$ system [SAP (Suisse) SA, Bienne, Switzerland]. We defined 1 bed day as one bed occupied by one patient during 1 day and one night.

\section{Data analysis}

We transformed the dataset using Python ${ }^{\mathrm{TM}}$ 2.7.6 (Python Software Foundation, Beaverton, OR, USA). We analyzed the data with the software R (R Core Team (2018). $\mathrm{R}$ foundation for Statistical Computing, Vienna, Austria) version 3.4.4 (Integrated Development for R. RStudio, Inc., Boston, MA, USA). We used the R packages fpp and forescast for all the time series analyses. $\mathrm{R}$ was also used for data edition and presentation.

\section{Study measurements}

We measured drug prescription periods of meropenem, voriconazole and caspofungin as DOTs [8]. We aggregated DOTs per month and weighted DOTs for the number of bed days (DOTs/1000 bed days). In addition, we counted the number of prescriptions, defined as one uninterrupted prescription period of a drug, independently of the duration of each prescription. We aggregated the number of prescriptions per month and weighted them for the number of bed days (prescriptions/1000 bed days). In addition to the days and number of prescriptions of the three drugs, age, gender and length of stay were matched with prescription data.

\section{Longitudinal analysis of prescription data}

We modeled the time trend and the impact of the alert on two outcome measures. Firstly, using interrupted time series regression, we assessed whether and in which magnitude the DOTs/1000 bed days changed after introduction of the alert. Secondly, we assessed whether and in which magnitude the number of prescription/1000 bed days changed after introduction of the alert. For both outcomes, we analyzed prescriptions of all three drugs together as well as the prescriptions of each drug independently.

\section{Seasonality}

In order to account for the seasonal effect on drug prescriptions, we removed the seasonal component of the 
time series as follows: first, we performed a decomposition of the time series, i.e. the monthly prescription periods, into the trend, the seasonal (periodic, additive) effect and the remaining random effect. For this purpose, we determined the trend of the original data using the $m a$ (simple moving average smoother) function of the R-package forecast (Fig. 2A). Second, we subtracted the trend from the original data (Fig. 2B). Third, we then calculated the mean of each month of the remaining data to estimate the seasonal effect (Fig. 2C). Thereafter, we removed the seasonal effect from the original data to assess the trend with exclusion of seasonality (Fig. 2D). The R-package ts (time series) was used for all calculations.

\section{Interrupted time series regression}

To analyze the potential impact of the electronic alert on prescription periods, we analyzed the time trend of prescriptions of meropenem, voriconazole and caspofungin by using interrupted time series regression analysis. The analysis of the potential impact of the electronic alert was performed on the data with the seasonal effect removed. For this, we modelled the prescriptions before and after the introduction of the alert on July 1, 2011, by allowing a change in intercept and slope at this day. In particular, we used the segmented regression model

$$
\begin{aligned}
& \text { prescriptions } \sim b_{0} * \text { month }+b_{1} * \text { intervention } \\
& \quad+b_{2} * \text { month } * \text { intervention, }
\end{aligned}
$$

where month indicates the month (numerical variable from 1 to 72 ) and intervention is a binary variable indicating whether the alert was present when the drug was prescribed or not. The coefficient $b_{1}$ is the intercept and represents the immediate change in prescriptions after the introduction of the alert. The coefficient $b_{2}$ is the change in slope of the regression after the introduction of the alert and represents the evolution of prescriptions over time.

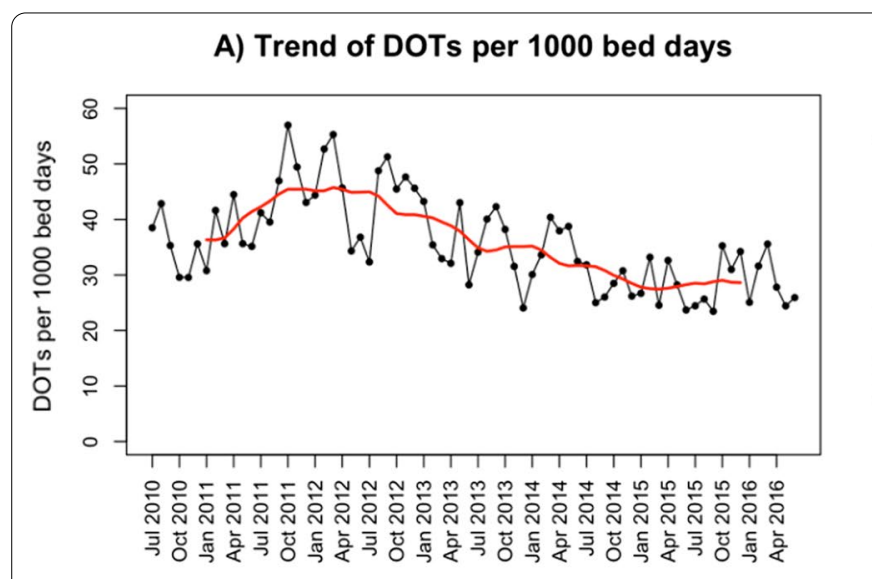

C) Seasonal effect

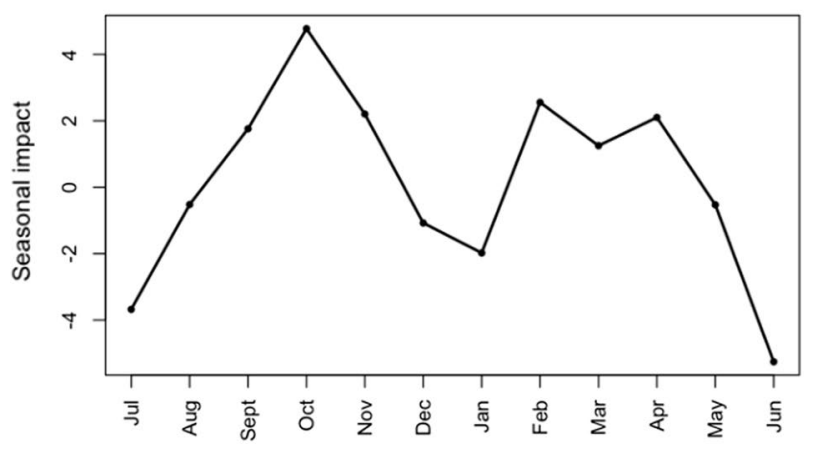

B) Removing the trend from the original data

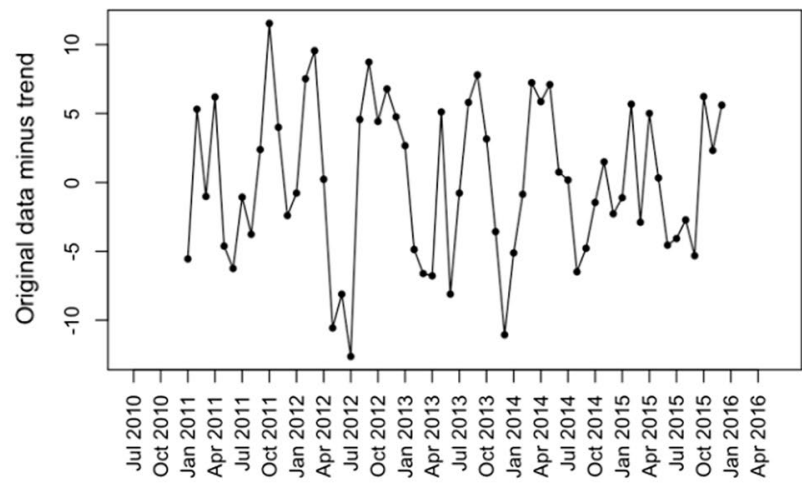

D) Trend of the original data without season

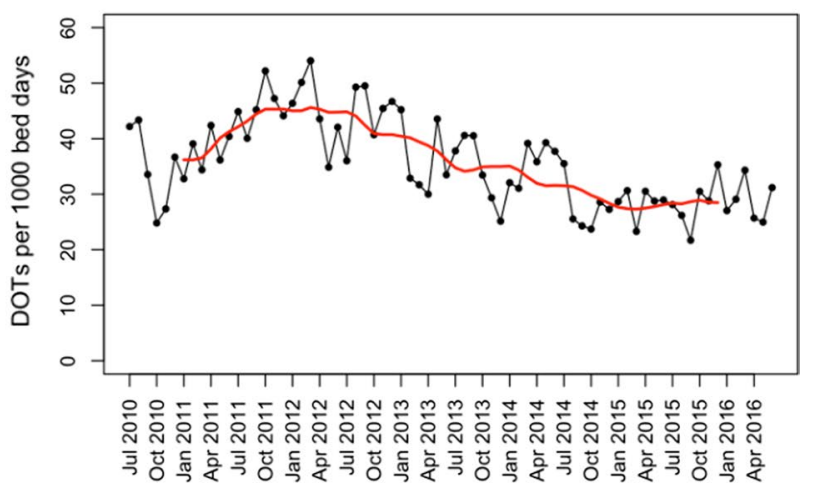

Fig. 2 A The trend (red) of the original data: in this example days of therapy (DOTs) per 1000 bed days. B The original data (DOTs per 1000 bed days) minus the trend. C The seasonal effect was calculated based on the original data with the trend removed. D The seasonal effect was subtracted from the original data to obtain the trend (red) which is not influenced by seasonality 


\section{Results}

\section{Study population}

From July 1, 2010, until June 30, 2016, we obtained data covering a total of $1,771,543$ bed days for all hospitalized patients, with a median of 24,364 bed days per month (IQR 22,901-25,653). The 4100 cases with prescription of one or more of the three substances accounted for 121,259 bed days in our institution (Table 1 ).

The median length of stay (LOS) was 23 days [interquartile range (IQR) 12-38]. The LOS was longest with 35 days for patients who were prescribed caspofungin (IQR 19-57), followed by meropenem with 24 days (IQR 14-40) and voriconazole with 24 days (IQR 9-36). The median age at admission was 57 years (IQR 43-66). $42.2 \%$ of patients who were prescribed one or more substances were female, with similar proportions across each of the three substances.

\section{Descriptive analysis of DOTs and number of prescriptions} From July 1, 2010, until June 30, 2016, we counted 5549 prescriptions and 64,281 DOTS during 4100 hospital stays (Table 1). Meropenem accounted for $60.9 \%$ of all DOTs (39,156 DOTs, 3695 prescriptions), followed by voriconazole with 19.7\% of all DOTs (12,640 DOTs, 1055 prescriptions) and caspofungin with $19.4 \%$ of all DOTs (12,485 DOTs, 799 prescriptions). The median duration of a prescription was 9 (IQR 5-15) days (caspofungin, 11 (IQR 6-18) days; meropenem, 8 (IQR 5-14) days; and voriconazole, 8 (IQR 4-18) days). In 3344 of 4100 cases $(81.6 \%)$, only one substance was prescribed (meropenem alone in 2558 cases, voriconazole in 570 cases, and caspofungin in 216 cases). In 663 cases (16.2\%) two substances were prescribed (meropenem and voriconazole in 271 cases, meropenem and caspofungin in 349 cases, voriconazole and caspofungin in 43 cases) and in 93 cases $(2.3 \%)$ all three substances were prescribed simultaneously.
Overall, the median number of DOTs per 1000 bed days (including all hospitalized patients) per month was 34.7 (IQR 29.6-41.8) with a minimum of 23.5 in September 2015 and a maximum of 57.0 in October 2011 (Fig. 3A). The median DOTs/1000 bed days per month was 21.1 (IQR 17.9-25.5]) for meropenem prescriptions, 7.0 (IQR 5.2-8.6) for voriconazole and 5.6 (IQR 4.0-9.4) for caspofungin. The median number of prescriptions/1000 bed days was 3.1 (IQR 2.7-3.4) with a minimum of 2.0 in September 2015 and a maximum of 4.2 in October 2011 (Fig. 3B).

We observed a median of 2.1 (IQR 1.7-2.4) prescriptions/1000 bed days for meropenem, 0.6 (IQR 0.5-0.7) for voriconazole and 0.4 (IQR $0.3-0.5$ ) for caspofungin.

\section{Interrupted time series regression DOTs/1000 bed days}

There was no significant change $(-0.58,95 \%$ CI $-1.48-0.33, P=0.2)$ in the trend of prescriptions (slope of DOTs) when comparing the time before the alert, i.e., July 2010 until June 2011, with the time after the alert, i.e., July 2011 until June 2016 (Table 2 and Fig. 4).

When analyzing prescriptions of the three drugs of interest separately, only DOTs/1000 bed days for voriconazole revealed a significant decrease in slope $(P=0.0017)$ after the introduction of the alert. Noteworthy, the regression intercept was significantly higher than $0(P=0.01)$ when analyzing DOTs/1000 bed days for all drugs combined, as well as for meropenem $(P=0.0014)$ and caspofungin $(P<0.001)$, meaning that prescriptions of these drugs increased significantly immediately after introduction of the alert. Only in the case of voriconazole, the intercept was significantly lower than $0(P<0.001)$ after the introduction of the alert.

Table 1 Prescription data and study population

\begin{tabular}{|c|c|c|c|c|}
\hline & Total & Meropenem & Voriconazole & Caspofungin \\
\hline \multicolumn{5}{|l|}{ Duration and number of prescriptions } \\
\hline Number of DOTs & 64,281 & 39,156 & 12,640 & 12,485 \\
\hline DOTs per prescription period, median [IQR] & $9[5,15]$ & $8[5,14]$ & $8[4,18]$ & $11[6,18]$ \\
\hline Number of prescription periods & 5549 & 3695 & 1055 & 799 \\
\hline \multicolumn{5}{|l|}{ Study population } \\
\hline Episodes & 4100 & 3271 & 977 & 701 \\
\hline Length of stay, days & 121,259 & 103,078 & 27,141 & 31,246 \\
\hline Length of stay, days, median [IQR] & $23[12,38]$ & $24[14,40]$ & $24[9,36]$ & $35[19,57]$ \\
\hline Female sex, n (\%) & $1731(42.2)$ & $1367(41.8)$ & $441(45.1)$ & $311(44.4)$ \\
\hline Age on admission, years, median [IQR] & $57[43,66]$ & $57[44,67]$ & $55[43,65]$ & $53[39,63]$ \\
\hline
\end{tabular}

DOTs days of therapy, IQR interquartile range 


\section{a) DOTs per 1000 bed days}

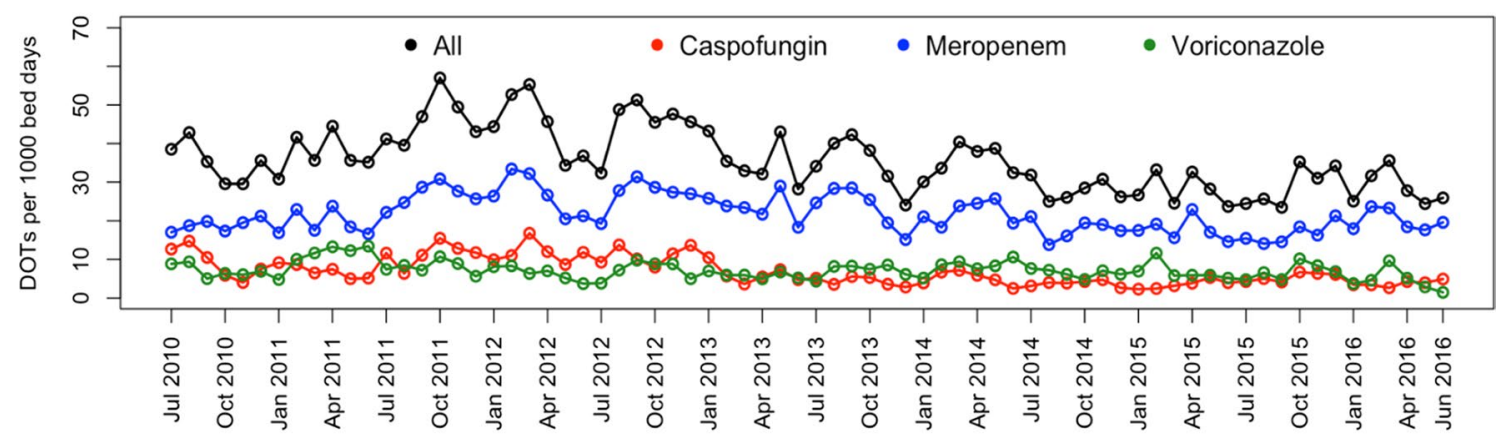

b) Number of prescriptions per $\mathbf{1 0 0 0}$ bed days

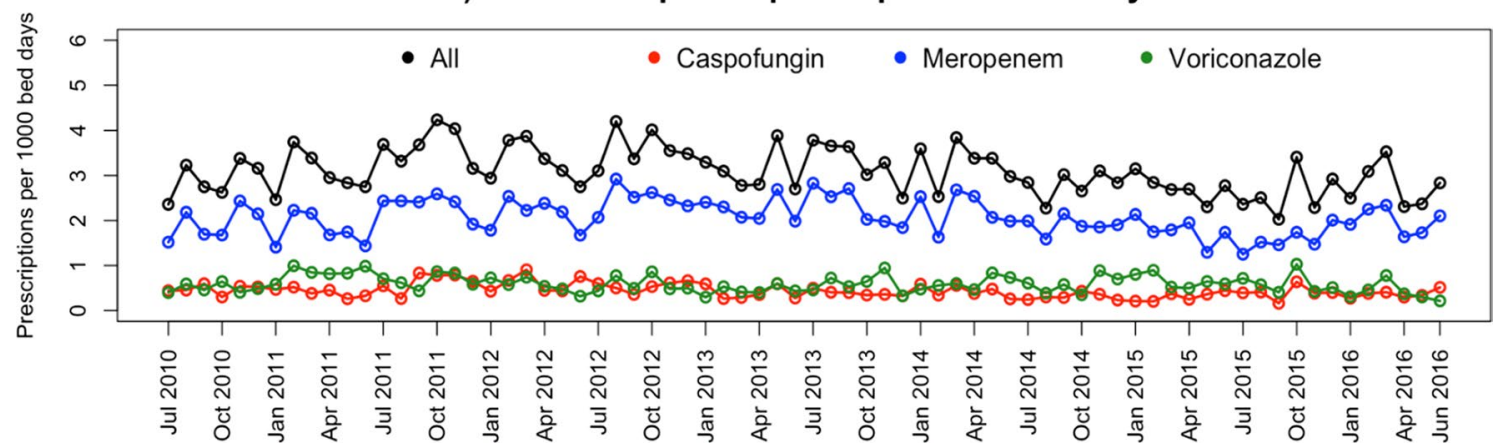

Fig. 3 A The total number of days of therapy (DOTs) per 1000 bed days, total and for the three drug classes separately; $\mathbf{B}$ number of prescription periods per 1000 bed days, total and for the three drug classes separately

Table 2 Results of the regression analysis of DOTs per 1000 bed days and number of prescriptions per 1000 bed days

\begin{tabular}{|c|c|c|c|c|}
\hline Variable & All drugs & Meropenem & Voriconazole & Caspofungin \\
\hline \multicolumn{5}{|c|}{ DOTs/1000 bed days [95\% Cl] } \\
\hline Intercept pre-interv. & $37.38[30.76,44.0]$ & $20.49[16.36,24.61]$ & $12.2[9.75,14.64]$ & $4.7[1.53,7.86]$ \\
\hline Change of intercept & $9.23[2.07,16.40]$ & $7.45[3.00,11.90]$ & $-4.61[-7.26,-1.96]$ & $6.39[2.97,9.82]$ \\
\hline P intercept & 0.0123 & 0.0014 & $<0.001$ & $<0.001$ \\
\hline Slope pre-intervention & $0.2[-0.7,1.1]$ & $0.2[-0.36,0.76]$ & $0.51[0.18,0.85]$ & $-0.51[-0.94,-0.08]$ \\
\hline Change of slope & $-0.58[-1.48,0.33]$ & $-0.39[-0.95,0.17]$ & $-0.55[-0.88,-0.21]$ & $0.36[-0.07,0.79]$ \\
\hline Pslope & 0.2079 & 0.1713 & 0.0017 & 0.1018 \\
\hline \multicolumn{5}{|c|}{ Prescriptions per 1000 bed days [ $95 \% \mathrm{Cl}]$} \\
\hline Intercept pre-interv. & $3.28[2.78,3.78]$ & $1.93[1.52,2.34]$ & $0.97[0.77,1.17]$ & $0.38[0.21,0.54]$ \\
\hline Change of intercept & $0.37[-0.17,0.92]$ & $0.52[0.08,0.97]$ & $-0.36[-0.58,-0.14]$ & $0.21[0.03,0.39]$ \\
\hline$P$ intercept & 0.1738 & 0.0211 & 0.0014 & 0.0204 \\
\hline Slope pre-intervention & $0.05[-0.02,0.12]$ & $0.01[-0.05,0.06]$ & $0.05[0.02,0.07]$ & $-0.01[-0.03,0.01]$ \\
\hline Change of slope & $-0.07[-0.13,0.00]$ & $-0.02[-0.08,0.04]$ & $-0.05[-0.08,-0.02]$ & $0[-0.02,0.03]$ \\
\hline Pslope & 0.0619 & 0.4724 & $<0.001$ & 0.7092 \\
\hline
\end{tabular}

$\mathrm{Cl}$ confidence interval, DOTs days of therapy, interv. intervention

\section{Prescriptions $/ 1000$ bed days}

Similar to the previous analysis, there was no significant change $(P=0.062)$ in slope when analyzing all drug classes combined. Again, only the slope of voriconazole prescriptions per 1000 bed days decreased significantly $(P<0.001)$ after the introduction of the alert (Table 2 and Fig. 5). Changes in the intercept were in line with the analysis of DOTs/1000 bed days. 
a) Days of therapy: all prescriptions

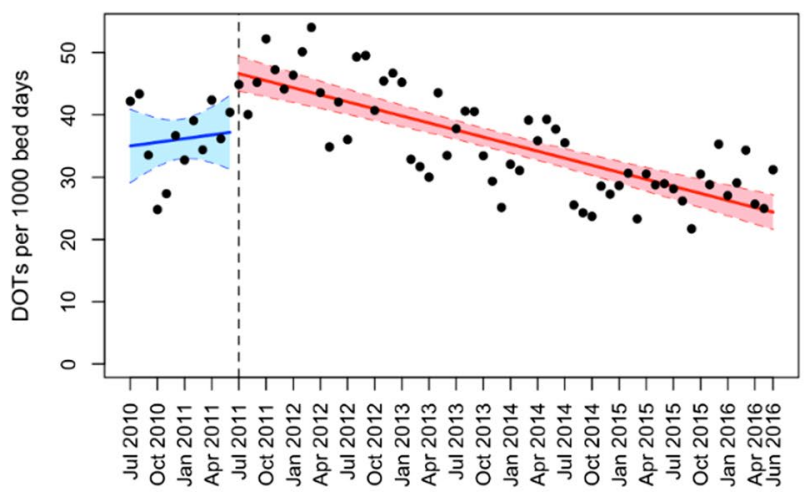

c) Days of therapy: Voriconazole

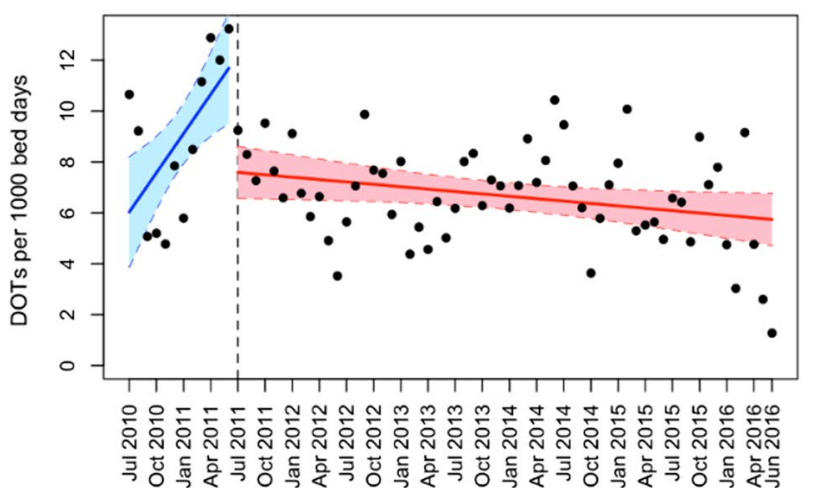

b) Days of therapy: Meropenem

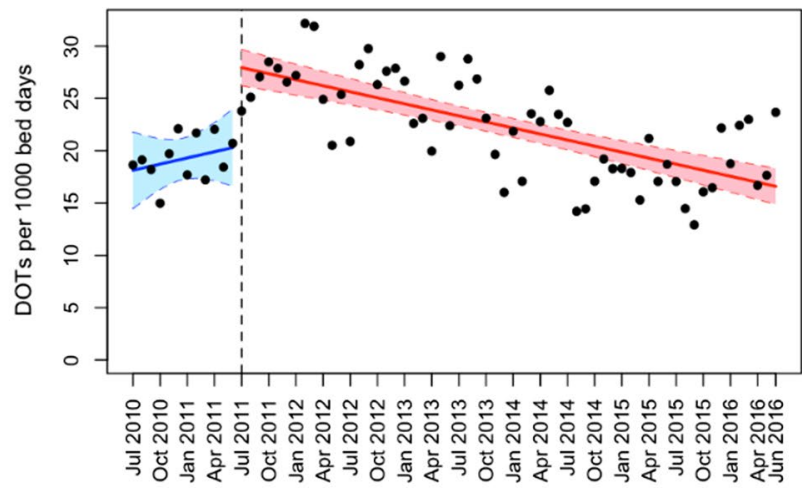

d) Days of therapy: Caspofungin

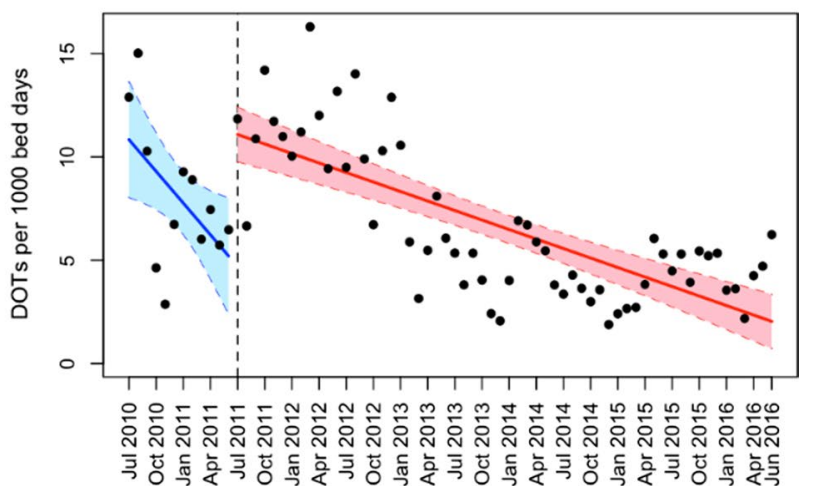

Fig. 4 Interrupted time series regression analysis of days of therapy (DOTs) per 1000 bed days, for all substances combined and for the three single substances separately

\section{Discussion}

This study examined the effect of an electronic alert on duration and number of prescriptions of anti-infective substances. Overall, the duration and the number of prescriptions tended to decrease after introduction of the alert, but the effect was not statistically significant. The stratified analysis of the individual drugs showed that only the prescriptions of voriconazole decreased significantly after the intervention.

The reduction of the duration as well the number of prescriptions of voriconazole is an encouraging signal supporting review of prescriptions by prescribers themselves [8]. Whether the alert alone, further non-measured interventions or a decreasing incidence in invasive fungal infections, led to this reduction, cannot be assessed due to the study design and the limited data available. Furthermore, the ASP at our institution is involved in many interventions, which possibly also explain the reduction of voriconazole prescriptions (ID consultations, ID visits on intensive care units, local guidelines, educational programs). Epidemiological reasons, such as increasing rates of multidrug-resistant gram-negative bacteria or an increasing incidence of invasive or azole-resistant $\mathrm{Can}$ dida infections, on the other hand, may have concealed a true intervention effect for meropenem or caspofungin $[11,17]$. As voriconazole is mainly used for the treatment of invasive Aspergillus fumigatus infections, such an opposite trend may not have played a major role for this substance.

Although the message that was provided in the alert clearly suggested to strongly re-consider the indication of the therapy, it may not have persuaded the treating physicians to stop the therapy or to contact the ID team. The direct effect of the alert on the number of ID consultations was not analyzed in this study but is an outcome of interest for further studies.

Our results are consistent with a previous publication from Lesprit et al. [18] In a similar study, the investigators could show that distribution of an information sheet advising an adaptation of treatment and a questionnaire at day 4 of an i.v. antibiotic therapy did not influence prescriptions of 13 selected i.v. antibiotics, in comparison 


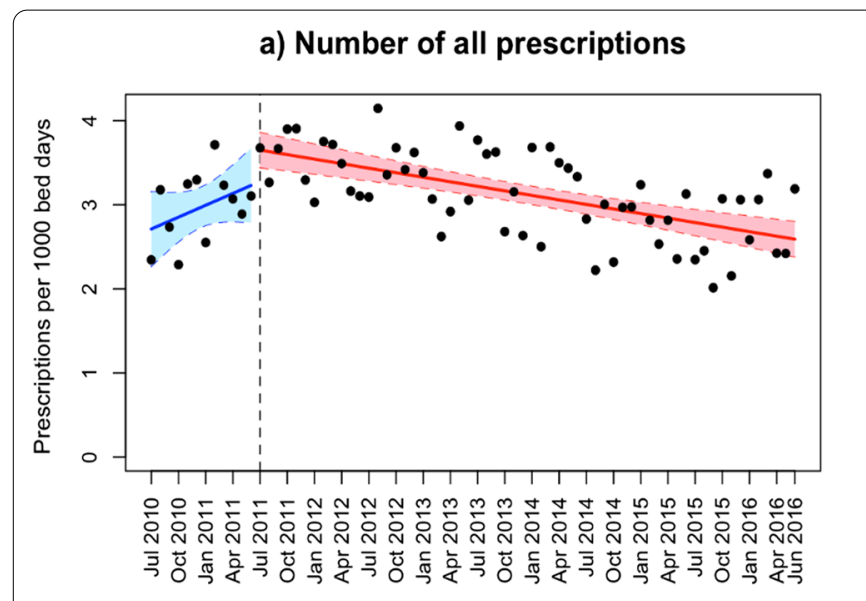

c) Number of prescriptions: Voriconazole

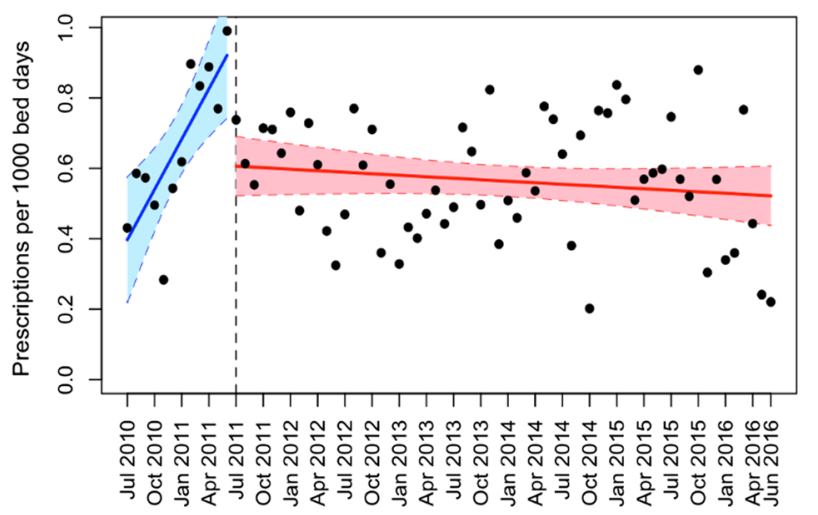

b) Number of prescriptions: Meropenem

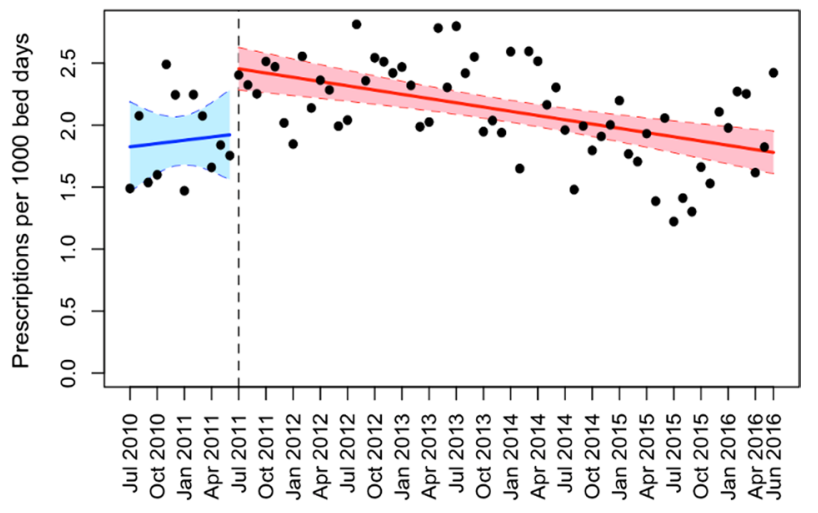

d) Number of prescriptions: Caspofungin

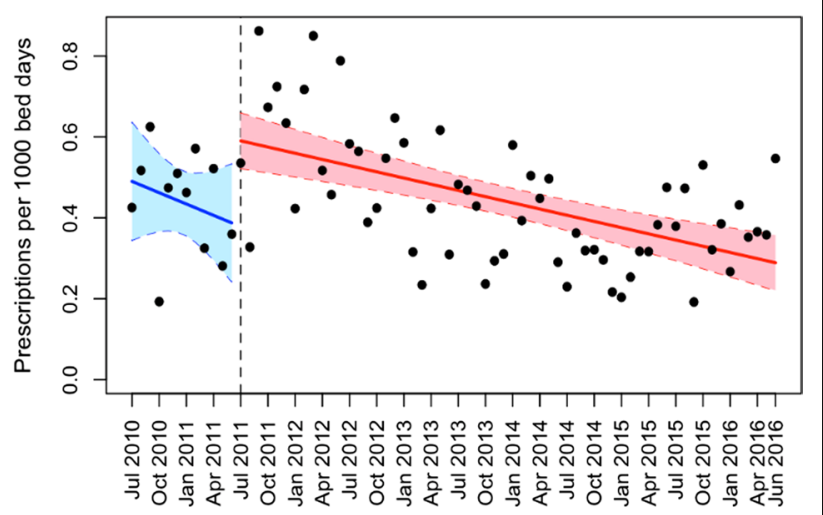

Fig. 5 Interrupted time series regression analysis of the number of prescriptions per 1000 bed days, for all substances combined and for the three single substances separately

to the control group. In the group with an additional ID consultation, prescriptions were significantly modified after day 4. Similarly, a previous study in the intensive care setting of a tertiary hospital in Chicago has shown a clear benefit of checklist and face-to-face prompting of physicians on antibiotic duration and risk-adjusted mortality in ICU patients receiving antibiotics, when compared to a checklist alone [19]. Prompting may be seen as part of prospective audit and feedback. Such interventions catalyze contacts between physicians and the ID advising team, which may be much more effective than self-stewardship [20, 21]. Prospective audit and feedback require available ID physicians and the resources for such a support are oftentimes lacking. ASP in our institution is based on ID consultation (on demand), ID visits in ICUs, and publications of guidelines, reports on consumption of anti-infective substances and bacterial resistance patterns.

An additional preauthorization by an ID physician, which is a more drastic intervention, would have possibly led to a stronger effect of the message provided [8]. However, the medical direction in Swiss hospitals rarely enforces preauthorization for antibiotics. This is possibly due to a strong prescriber autonomy in Switzerland. The absence of a patient, disease or substance specific education, further key interventions of ASPs, may also have led to the lack of statistical difference in this intervention study [8]. Furthermore, the psychological effect of the alert on the prescribing physicians may have vanished over time, as seen in other studies with electronic stewardship interventions, although such a pattern could not be confirmed in our data [22].

Finally, targeting only the prescribing physicians may have reduced the potential impact of the alert. Prescribing physicians are mostly fellows in training, who due to lack of time, motivation or knowledge, may underestimate the importance of the optimization of anti-infective prescriptions. One the other hand, the choice of the antiinfective substance is almost always made by the residents or specialists and rather not fellows. 
Strengths of this study are the large number of patients, the long observation period and the use of prescription data. The study has also several limitations. We cannot explain why we observed a sudden increase of meropenem and caspofungin prescriptions shortly after the alert was introduced. The message provided at and $72 \mathrm{~h}$ after prescription advised to re-evaluate the choice of the substance or to contact the ID team. The quality of the dataset depends on the electronic prescription system, which is in a constant modification state. It is possible that the alert introduction led to an optimization of data and thus captured more prescriptions. One other hypothesis is that the ID counselling service was more often involved, and indeed decided to lengthen or re-introduce prescriptions, since the chosen antibiotics seemed justifiable to the ID team. Third, we do not collect additional clinical data of included patients, which clearly exclude further explorative analyses. Also, structural changes have certainly occurred during the study period, which have not been assessed in this analysis but may well have influenced the results observed. Furthermore, data on other broad-spectrum antibiotics were not analyzed. This prevents us to assess whether the intervention led to switches to other broad-spectrum antibiotics, not covered by the alert and thus escaping our surveillance. Last, generalizability to other settings is limited since data originates from a single, tertiary care centre.

\section{Conclusions}

The introduction of an electronic reminder for prescriptions of meropenem, voriconazole and caspofungin led to a significant decrease of voriconazole prescriptions in comparison with the pre-intervention period, but did not consistently alter prescription patterns of meropenem or caspofungin. According to the results and other available evidence, self-stewardship supported by electronic reminders may only have a limited impact on prescriptions of anti-infective substances. Self-stewardship may thus benefit from face-to-face prompting with ID physicians and audit and feedback.

\begin{abstract}
Abbreviations
ASP: Antimicrobial stewardship program; ATC: Anatomical Therapeutic Chemical; DDD: Defined daily doses; DOTs: Days of therapy; ID: Infectious disease; IDSA: Infectious Diseases Society of America; IQR: Interquartile range; LOS: Length of stay; SHEA:The Society for Healthcare Epidemiology of America.
\end{abstract}

\section{Acknowledgements}

The authors thank the Medical Data Management Team for implementing the alert and retrieving data from electronic medical records.

\section{Authors' contributions}

The study was designed collaboratively by NE, CK, RW and SPK. Data collection carried out by LC, KK and DS. Data analysis and interpretation by LC, KK, DS, SEC, RK, RW and SPK. Manuscript preparation by LC, KK, NE, DS, SEC, CK, RK, RW and SPK. All authors read and approved the final manuscript.
Funding

No funding reported.

\section{Availability of data and materials}

The datasets generated during and analysed during the current study are not publicly available due to confidentiality but are available from the corresponding author on reasonable request.

\section{Declarations}

\section{Ethics approval and consent to participate}

This observational trial complies with the current version of the Declaration of Helsinki and the national legal and regulatory requirements and has been approved by the Canton Ethics Committee (Kantonale Ethikkommission Zurich, Switzerland, KEK-ZH-Nr 2011-0031/1 and PB_2018-00032). Since data used were anonymous and in compliance with the Swiss legislation governing human research (Federal Act on Research Involving Human Being, Human Research Act [HRA] of September 30, 2011, Status as of January 1, 2014, Art. 2 Abs. 2 lit. c), the Canton Ethics Committee waived the need for informed consent (Kantonale Ethikkommission Zurich, Switzerland, KEK-ZH-Nr 2011-0031/1 and PB_2018-00032).

\section{Consent for publication}

Not applicable.

\section{Competing interests}

The authors declare that they have no competing interests.

Received: 11 August 2021 Accepted: 15 December 2021

Published online: 20 December 2021

References

1. Marshall BM, Levy SB. Food animals and antimicrobials: impacts on human health. Clin Microbiol Rev. 2011;24(4):718-33.

2. Fair RJ, Tor Y. Antibiotics and bacterial resistance in the 21 st century. Perspect Med Chem. 2014;6:25-64.

3. Meyer E, Gastmeier P, Deja M, Schwab F. Antibiotic consumption and resistance: data from Europe and Germany. Int J Med Microbiol. 2013;303(6-7):388-95.

4. Goossens H, Ferech M, Vander Stichele R, Elseviers M. Outpatient antibiotic use in Europe and association with resistance: a cross-national database study. Lancet. 2005;365(9459):579-87.

5. Fishman N, Society for Healthcare Epidemiology of America, Infectious Diseases Society of America. Policy statement on antimicrobial stewardship by the society for healthcare epidemiology of America (SHEA), the infectious diseases society of America (IDSA), and the pediatric infectious diseases society (PIDS). Infect Control Hosp Epidemiol. 2012:33(4):322-7.

6. DiazGranados CA. Prospective audit for antimicrobial stewardship in intensive care: impact on resistance and clinical outcomes. Am J Infect Control. 2012;40(6):526-9.

7. Elligsen M, Walker SA, Pinto R, et al. Audit and feedback to reduce broad-spectrum antibiotic use among intensive care unit patients: a controlled interrupted time series analysis. Infect Control Hosp Epidemiol. 2012;33(4):354-61.

8. Barlam TF, Cosgrove SE, Abbo LM, et al. Implementing an antibiotic stewardship program: guidelines by the infectious diseases society of America and the society for healthcare epidemiology of America. Clin Infect Dis. 2016;62(10):e51-77.

9. Dellit TH, Owens RC, McGowan JE Jr, et al. Infectious diseases society of America and the society for healthcare epidemiology of America guidelines for developing an institutional program to enhance antimicrobial stewardship. Clin Infect Dis. 2007;44(2):159-77.

10. Foolad F, Nagel JL, Eschenauer G, Patel TS, Nguyen CT. Disease-based antimicrobial stewardship: a review of active and passive approaches to patient management. J Antimicrob Chemother. 2017;72(12):3232-44. 
11. Schweizerische Eidgenossenschaft. Swiss antibiotic resistance report 2016. http://www.anresis.ch/files/pdf/BAG_Swiss_Antibiotic_Resistance_ Report_2016_WEB.pdf. Accessed 18 July 2018.

12. Meletis G. Carbapenem resistance: overview of the problem and future perspectives. Ther Adv Infect Dis. 2016;3(1):15-21.

13. McLaughlin M, Advincula MR, Malczynski M, Qi C, Bolon M, Scheetz MH. Correlations of antibiotic use and carbapenem resistance in enterobacteriaceae. Antimicrob Agents Chemother. 2013;57(10):5131-3.

14. Schwaber MJ, Carmeli Y. Carbapenem-resistant Enterobacteriaceae: a potential threat. JAMA. 2008;300(24):2911-3.

15. Kachalov VN, Nguyen H, Balakrishna S, et al. Identifying the drivers of multidrug-resistant Klebsiella pneumoniae at a European level. PLoS Comput Biol. 2021;17(1): e1008446.

16. WHO Collaborating Centre for Drug Statistics Methodology. Norwegian Institute of Public Health. ATC/DDD Index 2018 (last updated: 2017-1220). https://www.whocc.no/atc_ddd_index/. Accessed 20 June 2018.

17. Lamoth F, Lockhart SR, Berkow EL, Calandra T. Changes in the epidemiological landscape of invasive candidiasis. J Antimicrob Chemother. 2018;73(suppl_1):i4-13.

18. Lesprit P, Landelle C, Girou E, Brun-Buisson C. Reassessment of intravenous antibiotic therapy using a reminder or direct counselling. J Antimicrob Chemother. 2010;65(4):789-95.

19. Weiss $\mathrm{CH}$, Persell SD, Wunderink RG, Baker DW. Empiric antibiotic, mechanical ventilation, and central venous catheter duration as potential factors mediating the effect of a checklist prompting intervention on mortality: an exploratory analysis. BMC Health Serv Res. 2012;12:198.

20. Newland JG, Stach LM, De Lurgio SA, et al. Impact of a prospective-auditwith-feedback antimicrobial stewardship program at a children's hospital. J Pediatr Infect Dis Soc. 2012;1(3):179-86.

21. Cosgrove SE, Seo SK, Bolon MK, et al. Evaluation of postprescription review and feedback as a method of promoting rational antimicrobial use: a multicenter intervention. Infect Control Hosp Epidemiol. 2012;33(4):374-80.

22. Lee TC, Frenette C, Jayaraman D, Green L, Pilote L. Antibiotic self-stewardship: trainee-led structured antibiotic time-outs to improve antimicrobial use. Ann Intern Med. 2014;161 (10 Suppl):S53-58.

\section{Publisher's Note}

Springer Nature remains neutral with regard to jurisdictional claims in published maps and institutional affiliations.

Ready to submit your research? Choose BMC and benefit from:

- fast, convenient online submission

- thorough peer review by experienced researchers in your field

- rapid publication on acceptance

- support for research data, including large and complex data types

- gold Open Access which fosters wider collaboration and increased citations

- maximum visibility for your research: over 100M website views per year

At BMC, research is always in progress.

Learn more biomedcentral.com/submissions 